International Journal of Linguistics, Literature and Translation

ISSN: 2617-0299 (Online); ISSN: 2708-0099 (Print)

DOI: $10.32996 / \mathrm{ijllt}$

Journal Homepage: www.al-kindipublisher.com/index.php/ijllt

\title{
Speech Disfluencies in Simultaneous Interpretations of Spontaneous and Non- Spontaneous Speeches
}

\author{
Hicham Boughaba 8 (iD. \\ Professor of Linguistics and interpreting studies at King Fahd School of Translation Tangier, Morocco \\ $\triangle$ Corresponding Author: Hicham Boughaba, E-mail: hboughaba@uae.ac.ma
}

\begin{tabular}{|c|c|}
\hline ARTICLE INFORMATION & ABSTRACT \\
\hline Received: June 09, 2021 & \multirow{6}{*}{$\begin{array}{l}\text { The present paper is an attempt to examine speech disfluencies in simultaneous } \\
\text { interpretations of spontaneous and non-spontaneous speeches from English into } \\
\text { Arabic. It focuses on the difference between the rate of disfluencies in renditions of } \\
\text { spontaneous and non-spontaneous speeches. The data were collected from authentic } \\
\text { sessions of professional interpreters from English into Arabic. The speeches and } \\
\text { interpretations were transcribed. The data was divided into two different categories: } \\
\text { 'spontaneous source speeches and their renditions' and 'non-spontaneous speeches } \\
\text { and their renditions'. Disfluencies in the source and target texts were analyzed and } \\
\text { compared. The results of the analysis showed that the rate of disfluencies in } \\
\text { spontaneous speeches is significantly higher than the renditions of non-spontaneous } \\
\text { speeches. The analysis also demonstrated that silent pauses are the most frequent } \\
\text { disfluency in both categories of interpretations, followed by prolongations and filled } \\
\text { pauses. }\end{array}$} \\
\hline Accepted: July 10, 2021 & \\
\hline $\begin{array}{l}\text { Volume: } 4 \\
\text { Issue: } 7\end{array}$ & \\
\hline DOI: 10.32996/ijllt.2021.4.7.3 & \\
\hline KEYWORDS & \\
\hline $\begin{array}{l}\text { Speech disfluencies, simultaneous } \\
\text { interpreting, spontaneous speech, } \\
\text { non-spontaneous speech. }\end{array}$ & \\
\hline
\end{tabular}

\section{Introduction}

The ultimate goal of interpreting is to guarantee mutual understanding between speakers of different languages. In this regard, fluency is a key component of the interpreter's performance. A smooth flow will not only enhance the quality of the delivery but also upgrade the experience of the recipient of the interpretation. However, simultaneous interpreting is a highly complex verbal practice in which the interpreter must listen and process the source text then reformulate information segments into the target language. All these tasks must be fulfilled simultaneously which may hinder the fluency of the interpreter.

During their delivery, interpreters produce different types of disfluencies such as pauses, hesitations, and repetitions, which compromise their fluency. However, the recurrence of disfluencies may depend on several factors including the level of fluency in the source speech itself. Accordingly, the main focus of this paper is to shed light on disfluencies in the output of interpreters working from English into Arabic. It aims at exploring those disfluencies in interpretations of spontaneous and non-spontaneous speeches and drawing a comparison between them. By understanding the disfluencies in simultaneous interpreting, it becomes possible to broaden our knowledge of the processes of this type of interpreting, as well as to identify the challenges and obstacles faced by interpreters in the simultaneous mode.

The present paper is based on data collected from authentic sessions of professional interpreters from English into Arabic. The speeches and interpretations were transcribed. The data was divided into two different categories: 'spontaneous source speeches and their renditions' and 'non-spontaneous speeches and their renditions'. Disfluencies in the source and target texts were analyzed and compared.

\section{K C AL-KINDI CENTER \\ $\mathbf{R}$ D FOR RESEARCH AND}

Your gateway to world-class research

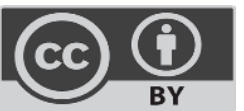

Published by Al-Kindi Center for Research and Development, London, United Kingdom. Copyright (c) the author(s). This open access article is distributed under a Creative Commons Attribution (CC-BY) 4.0 license 


\section{Review of the Literature}

\subsection{Speech Disfluencies}

\subsubsection{Defining Speech Disfluencies}

Speech disfluencies are a normal aspect of spoken language production. When talking, people often find themselves hesitating, pausing, repeating the same word, or starting their utterance all over; these failures in maintaining a smooth flow are a natural part of typical speech production. "Disfluencies occur at an average rate of around 6 per 100 fluent words" (Bortfeld et al, 2001, cited in Eklund, 2004, p. 216). However, linguists have neglected this phenomenon for a long time, since their main interest was focused on the competence of speakers to apply the linguistic rules correctly rather than their performance in the real world. Chomsky (1965), for example, sees disfluencies as 'errors' that reflect the incapacity of speakers to employ their language knowledge in their real-life performance (Eklund, 2004, p. 88). Moreover, the apparent irregularity and sporadic occurrence of disfluencies in typical speech have narrowed the importance of their study in the linguistic realm for a long time.

There is a lack of consensus over the definition of speech disfluencies since scholars have examined the phenomenon in different disciplines and from different perspectives. Gozy (2007) explains that "Speech disfluencies are generally defined as phenomena that interrupt the flow of speech and do not add propositional content to an utterance. There are various forms of disfluencies, and these might show some slight differences across languages." (Gozy, 2007, p.93). In the same respect, Ferreira and Bailey define speech disfluencies as "Any deviation in speech from ideal delivery" (Ferreira and Bailey, 2004, p. 231). Chafe (1994) suggests that speech disfluencies are evidence for nonconformity between what one is conscious of and what one says. He clarifies that "People often have trouble 'putting thought into words' and may believe that they have not adequately stated what they 'had in mind'" (Eklund, 2004, p. 103).

\section{Classification of disfluencies}

Speech disfluencies have received interdisciplinary attention. As a result, various scholars have established their classifications of the types of disfluencies and adopted different terminology. Lickley (2015) specifies that speech disfluencies are categorized from two different perspectives, a formal classification, based on the form words and syntactic units of the disfluencies, and a functional classification, which accounts for the link between the disfluencies and speech production processes.

\section{a. Formal classification}

Many scholars have attempted to classify the disfluencies according to the form they take within the utterance; in fact, the first work addressing this issue was done from a form perspective. This is the case with Mahl's classification of speech disturbances in 1956. Since then, other scholars have distinguished different forms of disfluencies (eg. Shriberg (1994); Heeman (1997); Lickley (1998)).

These scholars seem to agree on the following formal taxonomy:

- $\quad$ Repetitions (of part-words, words, phrases)

- Substitutions (the speaker replace a part-word, word, or string of words with another)

- $\quad$ Filled pauses (um, uh)

- Insertions (the speaker repeats an utterance, but adds a word or more)

- Deletions (the speaker stops an utterance mid-stream)

\section{b. Functional Classification}

The functional categorization of speech disfluencies is based on the work of Levelt's errors-based model of speech production. In his study concerning errors related to self-repairs during speech planning, Levelt distinguishes between covert errors and overt errors. A Covert error is "an error that has been made at some point in the planning process but has been detected and corrected by the speaker before it is articulated" (Levelt, 1989, p. 55). The speaker's covert error is corrected by a 'C repair', which will be manifested by a speech disfluency such as editing terms (like uh) or repetition of one or more lexical items. According to Postma and kolk (1993), speech disfluencies emanating from covert errors also include filled and silent pauses, repetition of part words, whole words and strings of words, prolongation sounds, and abrupt interruptions.

Recent studies of speech disfluency classification have been influenced by Levelt $C$ re-pairs disfluencies; among them, there is the following taxonomy adopted by Eklund (2004), Besser \& Alexanderson (2008), and others. 


\section{Unfilled pauses}

According to Gósy (2007), examining unfilled pauses is very problematic as it is hard to determine what pause can be perceived as an unfilled one. Long pauses are easily detected and can be easily explained, depending on their distribution inside the utterance. However, it is the lower limit of pauses that raises an issue. Many scholars have explored the minimal duration of unfilled pauses by employing a 'threshold' or a 'cut-points, where they set a cut-off point of $200 \mathrm{msec}$ for example and exclude all pauses below that duration. This is why some disfluency studies do not consider this notion altogether, despite its importance according to Martin (1970), who states: "of the hesitation phenomena, unfilled pauses are the most frequent" (Martin as cited in Eklund, 2004, p. 160).

\section{Repairs}

Repairs occur when the speaker notices that there is an error in his utterance, and he tries to repair himself before finishing the words or phrases. Fox et al. (2002) define this phenomenon as "the process by which speakers of a language stop, abort, repeat, or alter their turn before it comes to completion". Along the same line, Eklund (2004) suggests that a repair process may sometimes involve other notions, such as repetitions, substitutions, insertions, deletions.

\section{Prolongations}

Prolongations occur when the speaker lengthens a world more than the normal pace of his speech. Lickley (1994) suggests that speakers often prolong a syllable when they hesitate, to gain time, or to accommodate short-term difficulties in the retrieval of word forms. Eklund defines this notion as "phones that are longer than should be expected in normal-paced, fluent, speech." (Eklund, 2004).

\section{Explicit Editing Terms}

Sometimes speakers explicitly indicate that they are repairing themselves using words such as oops, sorry, I mean....; his notion is often referred to as Explicit Editing Terms. Shames and Sherrick (1963) assert that "speakers very often 'compose', 'edit' and 'prompt' themselves for their verbal behavior" (Shames \& Sherrick, 1963 as cited in Eklund, 2004, p 163).

\section{Filled Pause}

Filled pauses' occur when the speaker cannot maintain the flow of speech and introduces sounds such as 'uh' 'umm' within his utterance instead of silence. "When speakers cannot formulate an entire utterance at once, they may suspend their speech and introduce a pause or filler before going on." (Clark and Wasow, 1998, p 201). Lickley (1994) notes that filled pauses are among the easiest disfluencies to be detected because they can be easily distinguished from fluent words; this is why they have received greater attention within the study of speech disfluencies. It is also worth noting that they can take various forms depending on the language.

\section{Repetitions}

Speakers use repetition for various reasons; they can repeat a word or a phrase for emphasis or other rhetorical purposes. However, repetition can sometimes reflect a rupture in fluent speech. Lickley (2015) notes that "When speakers pause in the middle of saying something, then start again; they often restart by backtracking by one or two words and repeating them with a fluent continuation." (Lickley, 2015, p 459). Thus, repetition can signal the hesitation of the speaker. We can distinguish between the two types of repetition by taking into consideration their prosody. Lickley asserts that repetition for rhetorical effect, for example, is characterized by 'emphatic pitch, and produced in an uninterrupted sequence', while a hesitant repetition is often accompanied by a filled or unfilled pause. We can also recognize a hesitant repetition by using the context and the distribution within the utterance.

\section{Speech Disfluencies in Arabic}

The available work involving speech disfluency in Arabic belongs to different disciplines such as speech pathology and computational linguistics. There seems to be no study dealing with the exploration of disfluencies in a typical speech in Arabic. In 2009, a study was done by Abdella and al, addressing the relation between function and content words dichotomy and disfluencies among adult stutterers; these disfluencies included part-word repetitions, sound prolongations, and tense pauses. According to this study, the participants exhibited no important difference in disfluencies occurring in bare content and bare function words; however, a significantly higher amount of disfluencies was found in content-function words. This can be explained by a unique aspect of Arabic grammar which is fusional morphology. It refers to the possibility to combine word forms, including content and function words. This can lead to word forms that are characterized by a complex grammatical and morphological structure. (Abdalla et al, 2010).

In the field of computational linguistics, a recent study was conducted by Bahou et al, who explored disfluency and Out-OfVocabulary word processing in Arabic speech understanding. The disfluencies covered in this study were Repetitions, Omissions, Self-corrections, Restarts, and Word-fragments. The corpus contained 50 participants and 7590 utterances. After transcribing the 
study corpus, the examination of the utterances showed that $25.24 \%$ contained disfluencies (950 repetitions, 825 self-repairs, and 171 word-fragments). (Bahou et al, 2009)

The literature addressing speech disfluency in Arabic is very scarce. However, taking a brief look at the above-mentioned work can give us a slight idea about the work done previously, especially the types of disfluencies that we may find in Arabic speech.

\section{Speech Disfluencies in SI}

In simultaneous interpreting, fluency is regarded as an important quality that the interpreters must observe in their output. However, the complicated task of listening, understanding, and reformulating into the target language may jeopardize the smooth delivery of interpreters, which, in turn, reflects poorly on their overall performance. Despite its obvious importance, the issue of fluency received little attention from scholars; disfluencies occurring while interpreting has not yet been fully examined, and we still don't have an insightful vision of the many variables which interfere in the occurrence of speech interruptions among interpreters. Do they depend solely on the interpreter or does the source speech play a role in their occurrences? One would think that the fluency of the interpreter is determined mainly by his tendencies and competencies and is not influenced by the source speech; however, fluency of the speaker does play a significant role in the understanding of the interpreter; a speaker who pauses a lot, hesitates or randomly repeats his utterances will be much harder to understand than a speaker who maintains a smooth delivery. Any difficulty to understand the speaker and his intentions will interfere negatively with the mental process of the interpreter, which will be reflected in his rendition.

One of the earliest works on speech disfluency in simultaneous interpreting is a study conducted by Pochhacker in 1995 in which he compared the slips of tongues occurring in original speeches in a three-day conference with slips of tongues occurring in the interpreters' outputs. The study covered the following speech disfluencies: corrected and uncorrected slips and structure shifts (false starts, syntactic blends, and lexical blends). Pochhacker (1995) hypothesized that the slips of tongues in the interpreters' renditions would exceed the speaker's slips and contain more incorrect slips. He found that slips and shifts occur more frequently in the interpreters' output than in the original speech, except in the case of uncorrected slips. The study also demonstrated that false starts recur in the interpreters' and speakers' outputs in similar proportions.

A recent study by Maria Bakti in 2008 examined the speech disfluencies in the output of trainee and professional simultaneous interpreters from English into Hungarian. The study examined the renditions of 10 interpreters ( 7 trainees, 3 professionals) and covered error types disfluencies (ETD) including restarts, anticipation, grammatical errors, false word activation (Gozy dichotomy). The results revealed a similarity between the frequency of ETD among trainee and professional interpreters. The most frequently occurring ETD was: restarts, followed by grammatical errors and false word activation. Bakti concluded that the frequency of these ETD reflects difficulty with morphological and syntactic planning and coordination between lexical access and articulatory planning during speech production.

In 2000, Benedetta Tissi conducted a study in which she analyzed the interpretation of two tape-recorded spontaneous speeches from German into Italian. She worked with the non-fluencies category which includes silent pauses and disfluencies such as prolongation, false start, and hesitation... The study involved 10 native-Italian interpreting students. In addition to the analysis of non-fluencies in the interpreters' output, Tissi drew a comparison with the non-fluencies that occurred in the original speech. She concluded that the occurrence of non-fluencies in the interpreters 'output (TT) and the source text (ST) are related, the pauses in TT are longer and fewer than in ST, The TT contains more prolongations than ST, and filled pauses do not exhibit any pattern due to the individual differences between the participants. Tissi noted an important result concerning the communicative use of some non-fluencies, which she described as 'sometimes even strategic use', where the interpreters used tonic vowel prolongations to highlight a lexical item and used filled and silent pauses before a correction. (Tissi, 2000, p. 103)

\section{Methodology}

The phenomenon of speech disfluency in Arabic has not yet been explored. In an attempt to better grasp the concept of speech disfluency in simultaneous speeches, this paper examines the interpretation of two different types of speeches: spontaneous and nonspontaneous speeches. One of the major characteristics of spontaneous speech is the presence of interruptions such as pauses, hesitations, and repetitions, whereas non-spontaneous speech exhibits an even fluency thanks to the level of organization that preceded its delivery. This study aims at identifying and analyzing the disfluencies that occur while interpreting spontaneous and non-spontaneous speeches from English into Arabic and comparing them to investigate a possible correlation between the degree of the spontaneity of the speech and the quality of its interpretation. In attempting to address these issues, this paper will try to answer the following questions: What are the disfluencies appearing in the interpretations of spontaneous speeches? What are the disfluencies appearing in the interpretations of non-spontaneous speeches? How does the degree of the spontaneity of speeches affect the performance of interpreters? 
The present paper aims at identifying disfluencies in the output of interpreters from English into Arabic of spontaneous and nonspontaneous speeches; it attempts to draw a comparison between the interpreters' renditions in both cases. For this purpose, it is mandatory to examine the output of professional interpreters to limit the impact of disfluencies that emanate from a lack of experience and training. However, in the field of interpreting, it is very difficult to obtain data from professionals "to find authentic speeches and enlisting the cooperation of professional interpreters for research purposes" (Gile, 2005). To bridge this issue, the study will make use of preexisting, authentic interpreted speeches that are available online.

This research is an empirical exploratory study that intends to explore interpreters' disfluencies in two different contexts: spontaneous and non-spontaneous original speeches. Typically, exploratory research is concerned with answering questions of "what" and "how". Thus, this paper will explore what types of disfluencies occur in interpreting from English into Arabic and how they are affected by the disfluencies of the original speech. This paper is also a comparative study that intends to draw a comparison between the disfluencies occurring while interpreting a spontaneous speech vs a non-spontaneous speech.

The analysis of this study is both qualitative and quantitative; it intends to measure the rate of disfluencies in the output of interpreters and explore the underlying trends and features of these disfluencies.

To obtain solid data, the study used authentic performances of interpreters from different resources, namely existing interpretations of speeches that were broadcasted on Arabic TV Networks and an interpretation from a session of the general assembly of the United Nations. After selecting the interpretations, the original speeches were gathered to analyze their disfluencies as well.

The spontaneous speeches selected for this study are exclusively extracted from press conferences and interviews where the speaker had to answer a question immediately. This is to guarantee the spontaneity of the materials.

The total duration of recordings that were transcribed for this study amounts to:

Non-spontaneous speeches: 63:03 min

Rendition of non-spontaneous speeches: 61:92 min

Spontaneous speeches: $54: 79$

Rendition of spontaneous speeches: 56:05

The interpretations were all delivered by professional interpreters working for different TV Networks, namely Al-Jazeera and AlArabiya. One speech was interpreted by a professional working at the general assembly of the United Nations.

After selecting the material, the speeches were transcribed as faithfully as possible to preserve all features relevant to this study, as stated in Diriker (2004), "transcribing oral language ... involves representing oral language and its features such as hesitation, intonation, false starts, blends, semi- and non-verbal behavior in a form of writing that makes use of punctuation marks, layout, and symbols for representation." In this paper, the transcription adopted is a simplified one, where only the representation of the aspects, under focus in this study, were maintained; other features such as punctuation were dismissed to ensure the clarity of the other symbols used to track the disfluencies.

To detect and measure silent pauses, this study will make use of Audacity software, version 2.3.2 downloaded from https://www.audacityteam.org/download/. While this software helped with pauses detection, the measurement of pauses will be carried out manually.

Some speeches selected for this study are only available in video format on the internet, to convert the videos into Mp3 so that they can be processed by Audacity software, Freemake Video Converter software 4.1 .10 will be used. It is available on https://www.freemake.com/free_video_converter/.

For the quantitative analysis, the interpretations and original speeches were transcribed as faithfully as possible by listening to the recordings. Then, silent pauses were measured using Audacity software and their durations were indicated. Lastly, the rest of the disfluencies were counted and categorized according to the below-mentioned taxonomy positions. The positions of the disfluencies were indicated in the transcripts of both the source speeches and the interpretations. To conduct a qualitative analysis, samples of the original speeches and target speeches that contain significant disfluencies will be chosen to be described and analyzed.

This study focuses on the list of disfluencies mentioned below; it combines the taxonomy used by Eklund (2004), mentioned in the review of literature, and the taxonomy adopted by Tissi (2000). This classification is more detailed and exhaustive and will allow for clearer insight into disfluencies and speech production during simultaneous interpreting into Arabic. 
Unfilled pauses $P$

filled pauses $\mathrm{H}$

Vowel and consonant prolongations $\mathrm{L}$

Repetitions R

Restructuring/ Correction C

False start FS

Explicit Editing Terms $\mathrm{E}$

\section{Findings}

The results of this research demonstrate that disfluencies in spontaneous and non-spontaneous speeches are largely dominated by silent pauses. In spontaneous speeches, interpreters seem to have paused less than the speakers, which are thought to stem from the spontaneity of their delivery.

Furthermore, analysis of the frequency and duration of silent pauses in the interpreters' and speakers' deliveries show that the interpretations of spontaneous speech contain longer pauses than the source speeches. These results are in accordance with the findings of the research conducted by Tissi (2000), in which she compared the silent pauses in the speakers' and interpreters' performances from German into Italian.

In non-spontaneous speeches, articulatory and rhetorical silent pauses seem to enable the interpreter to keep a smooth flow in their deliveries, with less frequent pauses; they also seem to help interpreters anticipate the speakers' intentions. The second most frequent disfluency in both spontaneous and non-spontaneous speech renditions are prolongations, namely of the vowel $g$ and the consonant $J$ ' 'in the definite article ll. This frequency may either be attributed to the particularity of the Arabic phonetics or the strategic use of lightning in simultaneous interpreting, as it was pointed out previously, interpreters may resort to lengthening to be able to direct their attention towards listening or to fill the gap in their output while waiting for new information from the speaker.

Filled pauses and corrections in both spontaneous and non-spontaneous speeches are relatively frequent; the analysis, however, does not show any particular correlation between their distribution in the source and the target texts. In both categories, false starts are the lowest ranking type of disfluencies, which confirms findings by Tissi (2000).

In the renditions of the spontaneous speeches, interpreters seem to produce more repetitions than the source texts; however, it was noticed in the analysis of the data, that the repetitions of the speakers did not lead to repetitions of the interpreters, which may suggest that the latter were able to identify this type of disfluencies and omitted them in their output.

Concerning the correlations between the level of the spontaneity of the source texts and the fluency of the interpretations, the comparison of the data of the two categories: spontaneous and non-spontaneous renditions, indicates that interpreters of spontaneous speeches produce more speech disfluency than interpreters of non-spontaneous speeches. Judging by the results of this research, it seems that the frequency of interruptions in the spontaneous source texts does impact the rendition of the interpreters. On the other hand, interpretations of the non-spontaneous speech contain far less disfluency.

\section{Conclusion}

The present paper aimed primarily at examining the speech disfluencies in the output of simultaneous interpreters from Arabic into English. More precisely, it focused on the difference between the rate of disfluencies in renditions of spontaneous and nonspontaneous speeches. In an attempt to reach solid conclusions, data was collected from authentic renditions of professional interpretations.

The transcription of the speeches was simplified to include only the features relevant to this study. The analysis of the collected data was conducted base on classifications that were used in previous research. Apart from the comparison, the analysis also attempted to explore the common trends in the renditions of spontaneous and non-spontaneous speeches.

The analysis of the data yielded several conclusions. The major conclusion concerns the comparison between renditions of spontaneous and non-spontaneous speeches which led to conclude that the rate of disfluencies in spontaneous speeches is significantly higher than the renditions of non-spontaneous speeches, which distinctly showed the impact of disfluency in the 
source speech on the interpreter's performance. The analysis also demonstrated that silent pauses are the most frequent disfluency in both categories of interpretations, followed by prolongations and filled pauses.

The analysis did not manifestly demonstrate the influence of disfluencies' pattern in source speech on their distribution in the interpretations. On another note, further investigation is needed to explore the features of individual pattern which affect the use of speech disfluencies.

The research findings are reliable. However, this issue requires further similar studies to put these findings to the test. Future research would be much useful if it explored the individual tendencies of interpreters in producing speech disfluencies. In addition, an exploration of disfluencies in other modes of interpreting would be extremely enriching to the interpreting field.

\section{References}

[1] Abdalla, F, and Robb M. (2010) Stuttering and lexical category in adult Arabic speakers. Clinical linguistics \& phonetics. 24(10), pp 70-81.

[2] Bahou, Y and Belguith, L (2009) Disfluency and Out-Of-Vocabulary Word Processing in Arabic Speech Understanding. [Online]. Available from: https://www.researchgate.net/publication/238605642 Disfluency and Out-OfVocabulary_Word_Processing_in_Arabic_Speech_Understanding [Accessed 26/06/19]

[3] Bakti, M (2008). Speech Disfluencies in Simultaneous Interpretation. Available from:http://www.kuleuven.be/cetra/papers/papers.html [Accessed 26/06/19].

[4] Clark, H, and Wasow, T. (1998). Repeating Words in Spontaneous Speech. Cognitive psychology, 37(5), pp. $201-242$.

[5] Diriker, E. (2015). Simultaneous Interpreting. In F. Pochkacker, ed., Routledge Encyclopedia of Interpreting Studies, 1st ed. New York: Routledge, pp 382-384.

[6] Eklund, R. (2004) Disfluency in Swedish human-human and human-machine travel booking dialogues.Ph.D. thesis, Linköping Studies in Science and Technology, Dissertation No. 882, Department of Computer and Information Science, Linköping University, Sweden.

[7] Ferreira, F. and Bailey, K. (2004). Disfluencies and human language comprehension. TRENDS in Cognitive Sciences,8(5), pp. $231-237$.

[8] Gile, D. (1997) Conference interpreting as a cognitive management problem. In F.mPochhacker \& M. Shlesinger (Eds.), The interpreting studies reader. London: Routledge, pp. 163-176.

[9] Gile, D. (2005) Directionality in conference interpreting: A cognitive view. In R. Godijns, R and Hinderdael,M (Eds.), Directionality in interpreting. The 'Retour' or the Native

[10] Ghent: Communication and Cognition, pp.9-26

[11] Gósy, M. (2007) Disfluencies and Self-monitoring. Govor 26, pp 91-110.

[12] Kirchhoff, H. (1976) Simultaneous interpreting: Interdependence of variables in the interpreting process, interpreting models and interpreting strategies. In Pochhacker,F and Shlesinger,M (Eds.), The interpreting studies reader. London: Routledge. pp.111-119

[13] Levelt, W. (1989) Speaking. From Intention to Articulation. Cambridge MA: MIT Press.

[14] Levelt, W.(1999) Language production: a blueprint for the speaker. In C. Brown and P.

[15] Hagoort. (ed) Neurocognition of language. Oxford: Oxford University Press, pp. 83-122.

[16] Lickley, J R. (2015) Fluency and Disfluency. In M. A. Redford, ed., The handbook of speech production, 1st ed. West Sussex: John Wiley \& Son, pp. 445-473.

[17] Page, J. (2006) On Directionality in Interpreting. The Journal of Specialised Translation 6, pp.213-215

[18] Pöchhacker, F. (1995) Slips and Shifts in Simultaneous Interpreting. In Tommola, J. (Ed) Topics in Interpreting Research, (ed). Turku: The University of Turku, Centre for Translation and Interpreting, pp 73-90.

[19] Pöchhacker, F. (2015) Routledge encyclopedia of interpreting studies. New York: Routledge.

[20] Tissi, B. (2000) Silent pauses and disfluencies in simultaneous interpretation: A descriptive analysis". The interpreter's Newsletter, 10, pp.103126. 